\title{
The Acceleration Index as a Test-Controlled Reproduction Number: Application to COVID-19 in France*
}

\author{
Christelle Baunez $^{\dagger} \quad$ Mickael Degoulet ${ }^{\ddagger} \quad$ Stéphane Luchini, ${ }^{\S}$ \\ Matteo L. Pintus ${ }^{\top}$ Patrick A. Pintus॥ Miriam Teschl**
}

November 5, 2021

\begin{abstract}
We show that the acceleration index, a novel yet simple indicator that measures acceleration of viral spread (Baunez et al., 2021), corrects the reproduction number for the time-varying amount of tests. Not doing so results in the reproduction number being a biased estimate of viral acceleration and we provide a formal decomposition of the resulting bias. When applied to French data for the COVID-19 pandemic (from May 13 to November 19, 2020), our decomposition shows that the reproduction number consistently underestimates the resurgence of the pandemic since the summer of 2020, compared to the acceleration index which accounts for the time-varying volume of tests. Because the acceleration index aggregates all the relevant information and captures in real time the sizable time variation featured by viral circulation, it is a sufficient statistic to track the pandemic's propagation. As such it is a more accurate indicator to track the dynamics of an infectious disease outbreak in real time.
\end{abstract}

JEL Classification Numbers: I18; H12

Keywords: COVID-19; Reproduction Number; Lock-down; Acceleration Index; Real-time Analysis; France

\footnotetext{
*The authors thank the Editor and three referees for suggestions that prompted us to improve the paper. This work was supported by the French National Research Agency Grant ANR-17-EURE-0020, and by the Excellence Initiative of Aix-Marseille University - A*MIDEX.

†Senior Research Fellow, Aix-Marseille Univ, CNRS, Institut Neurosciences Timone. Email:christelle.baunez@univ-amu.fr

${ }^{\ddagger}$ Research Fellow, Aix-Marseille Univ, CNRS, Institut Neurosciences Timone. Email:mickael.degoulet@univamu.fr

§Research Fellow, Aix-Marseille Univ, CNRS, Aix-Marseille School of Economics. Email:stephane.luchini@univamu.fr

『AgroParisTech. Email:matteo.pintus@agroparistech.fr

"Professor, Aix-Marseille Univ, CNRS, Aix-Marseille School of Economics. Email:patrick.pintus@univ-amu.fr

**Associate Professor, Aix-Marseille Univ, CNRS, EHESS, Aix-Marseille School of Economics. Email:miriam.teschl@ehess.fr
} 


\section{Introduction}

The reproduction number is a widely used measure of how fast a pathogen propagates both at the outset and during an infectious disease outbreak (see for example May and Anderson [17]). One of its major shortcomings, however, is that it does not control for the quantity of tests (or any diagnostics) performed in real time. Doing so is of crucial importance for two reasons. One is the fact that accurate empirical estimates of reproduction numbers are time-varying in nature (see e.g. Fraser [11] among many others). A considerable source of time variation comes from the fact that the amount of tests changes substantially across time and hence affects the number of known cases, due to demand and supply effects. Second, testing acts as a magnifying lens on viral activity at least on the part of the population that has effectively been tested. The reproduction number however does not rely on that information but rather makes assumptions on the infectivity of positive cases based on the observation of onset of symptoms and transmission in closed systems such as households (see e.g. Cori et al. [9]). But this information, again, depends on tests (or any diagnostics more generally). Hence inferring infectivity from (assumed) transmissions is only secondary information based on the availability of tests. The logical consequence of these observations is that any measure of virus reproduction should take tests (or any available diagnostics) into account and this is exactly the issue we address in this paper.

In Baunez et al. [4, 5, 6], we have introduced an alternative and novel measure of viral spread in the context of COVID-19 - the acceleration index. This measure considers the variation of cases relative to the variation of tests and thus it avoids the shortcomings mentioned above. The purpose of this article is to discuss the reproduction number in the light of our acceleration index, and to show that the former is actually a special case of the latter and in fact a less accurate metric of the pandemic's time-varying speed.

We examine this important issue in two steps. In Section 2, we start from the very definition of the reproduction number as a gross rate of growth of infected people, traditionally denoted $R$, and derive a general formula that connects it to our acceleration index that we denote $\varepsilon$. The acceleration index is an elasticity that measures the proportional responsiveness of cases to tests, and it can also be thought of as the ratio between the current and average viral speeds. More specifically, we present an explicit measure of the ratio between $R$ and $\varepsilon$, the interpretation of which is further discussed in terms of the infectivity and test contributions. Our theoretical inquiry stresses that while the acceleration index is a ratio of growth rates - that of cases divided by that of tests - the reproduction number tracks only the growth rate of cases. In other words, the acceleration index corrects the reproduction number for the time-varying amount of tests. Not doing so results in the reproduction number being a biased estimate of viral acceleration and we provide a formal decomposition of the resulting bias.

In Section 3, we apply such an analysis to French data and we show that there is a sizeable difference between both measures. Indeed that the reproduction number $R$ largely under-estimates the spread 
of the virus, compared to our test-controlled measure of acceleration. In Appendix B, we provide a similar analysis for five other countries to show that this result is not limited to the French case. It is in this sense that we say that the reproduction number is biased. This has obviously important consequences if the reproduction number is used as the basis for which public health decisions such as entering or exiting a lock-down. We also look at the effects of the second lock-down period in France, which started October 30, 2020, through the lens of both indicators, as a further example that illustrates the bias unavoidably implied by not adjusting for the volume of tests over time when measuring the pandemic's acceleration.

\section{Materials and Methods}

About a century ago, a series of seminal articles by Kermack and McKendrick [12, 13, 14] have laid the foundations for a mathematical theory of epidemics. More specifically, their compartmental (that is, Susceptible, Infected and Removed or SIR-type) and time-since-infection models have been extensively used and refined in the academic literature about infectious and emerging diseases. A core concept in this paradigm is the reproduction number, usually noted $R$, which roughly captures how many secondary cases originate, on average, from a pool of primary cases who is still currently infectious (see May and Anderson [17]).

As evident from publications by health agencies around the world since the onset of COVID-19, much of the guidance for designing policy measures to curb the pandemic relies prominently on estimates of $R$, among other things. The reproduction number is initially a theoretical concept, conceived to understand the transmissibility of an epidemic. Many efforts have been put into defining ways to empirically estimate it. Broadly speaking, estimation strategies fall into two broad categories. The first one rests on the basic SIR model (see e.g. Weiss [22] for a clear exposition), which predicts that the reproduction number $R$ is the product of four parameters: the duration of infection, the number of contacts per case and the fraction of contacts who are in turn infected, on average, and finally the fraction of total population susceptible to infection. Although each of these parameters could be estimated in real-time, this turns out to be a gigantic task, in particular when a novel pathogen like SARS-Cov-2 emerges. A short-cut to avoid such a demanding procedure is to fit a SIR model using the number of cases, so as to estimate $R$ directly, given the infection duration (see, among many others, Althaus [1] for a recent example related to Ebola using maximum likelihood estimation). This is feasible, even in real-time, provided that enough data is available to ensure precision and structural assumptions about the time-dependency of $R$ are made. A caveat, though, is that such fitting procedures have limitations (see e.g. Cori et al. [10]). An additional issue arising from estimation based on compartmental models is the sizeable range of estimates. See Chris et al. [2] for SARS, and Viceconte and Petrosillo [20] for the early stages of COVID-19. The second estimation strategy addresses more directly the time-varying dimension of $R$, which is more in line with epidemiological and clinical data. Many health agencies rely on such estimates of time-since-infection 
transmission models rather than SIR-type models. Here the basic idea is that $R$ is essentially $(1+)$ the growth rate of infected, which is the ratio between the number of new (that is, secondary) cases arising, say, within 24 hours, and the number of primary cases (see Fraser [11]). For example, the French agency in charge of health statistics uses the Cori method, after Cori et al. [9] (see https://www.santepubliquefrance.fr/content/download/266456/2671953). Other European health agencies are also using this method, e.g. Austria (see https://www.ages . at/download/0/0/ e03842347d92e5922e76993df9ac8e9b28635caa/fileadmin/AGES2015/Wissen-Aktuell/COVID19/ Methoden_zur_Schätzung_der_epi_Parameter.pdf) and Germany (see https://www.rki.de/DE/ Content/InfAZ/N/Neuartiges_Coronavirus/Projekte_RKI/R-Wert-Erlaeuterung.pdf?__blob= publicationFile).

Our task here is to relate the acceleration index defined in Baunez et al. [5, 4] and the reproduction number that is estimated using the time-since-infection approach just described. The main purpose of this section is to derive a theoretical relationship between both concepts, which helps both to explain why they are different, to give a sense of the magnitude of their difference, and to state the conditions under which they are equivalent. We then turn, in the next section, to data to gauge whether the difference between the two matters to track the COVID-19 pandemic.

Suppose that data is available about the number of tested and positive persons, up to end date $T$. Denote $\left\{p_{1}, \ldots, p_{T}\right\}$ the historical times series of the new (per period) number of positive persons from date $t=1$ to end date $t=T$. Similarly, $\left\{d_{1}, \ldots, d_{T}\right\}$ is the historical times series of new (per period) diagnosed/tested persons. Denote $P_{t}=\sum_{\tau=1}^{t} p_{\tau}$ and $D_{t}=\sum_{\tau=1}^{t} d_{\tau}$ the cumulative numbers of positive and diagnosed persons up to date $t$.

As stressed in Baunez et al. [5], accurate information about the dynamics of a pandemic rests on both the number of cases and the number of tests, and the former cannot be properly understood without the latter. In that paper, we introduce an acceleration index, denoted $\varepsilon_{T}$ at date $T$, which is an elasticity that measures the proportional responsiveness of cases with respect to tests. Given that the number of cases and tests are not necessarily varying at the same rate across time, groups and also space, the acceleration index measures the percentage change of cases with respect to a percentage change of tests and is thus unit-free. The acceleration index is defined as follows:

$$
\varepsilon_{T}=\left[\frac{P_{T}-P_{T-1}}{P_{T}}\right] \div\left[\frac{D_{T}-D_{T-1}}{D_{T}}\right]
$$

Rearranging the terms of the latter equation, we see that the acceleration index relates to the daily and average positivity rates, in the following way:

$$
\underbrace{\frac{P_{T}-P_{T-1}}{D_{T}-D_{T-1}}}_{\text {daily positivity rate }}=\underbrace{\frac{P_{T}}{D_{T}}}_{\text {average positivity rate }} \times \underbrace{\varepsilon_{T}}_{\text {acceleration index }}
$$

Equation (2) shows that the acceleration index is an elasticity, which is a concept widely used by economists since Marshall [16], to study the responsiveness of demand with respect to a change in price of a good. The reason we call such an elasticity an index of viral acceleration can be clarified 
using an analogy with linear body motions. Given that our analysis relies on data about cases and tests only, with the latter as units of measurement, one can think of the acceleration index as the ratio between current and average viral speed. With tests as the unit-measurement, the daily positivity rate $p_{T} / d_{T}$ becomes a measure of current viral speed at date $T$, that is, the fraction of tested people that turn out to be positive on that day. The average positivity rate $P_{T} / D_{T}$ at date $T$ can be thought of as average viral speed, taken over the entire data sample. In Appendix A we illustrate through an example why we do not average over daily positivity rates in the usual way, but rather take the ratio of cumulated cases to cumulated tests. If then current viral speed is larger than average viral speed, we are in a situation of viral acceleration and the pandemic is on the loose. In that case, our acceleration index $\varepsilon_{T}$ is larger than one, which means that increasing tests by $1 \%$ leads to more than $1 \%$ of new cases. An arguably legitimate goal of public health policy would therefore be to make sure that the acceleration index gets smaller than one, i.e. that current viral speed becomes smaller than average viral speed: this would indicate that the pandemic decelerates and becomes under control. Ideally, one would like to find ever fewer cases the more one tests. This reasoning also shows why it is not sufficient to look at positivity rates alone - they only indicate viral speed. What matters for public health is to understand whether speed becomes greater or smaller compared to its historical average as tests increase, which is what our acceleration index measures. In Appendix A, we give an example using exponential growth, for which closed-form solutions are derived and can be used to further illustrate the interpretation of the acceleration index as a unit-free elasticity that relates to how the current viral speed compares to its historical average.

Regarding the reproduction number, our rather general assumption is, in accordance with the mathematical literature on epidemics, that the reproduction number is essentially a gross rate of growth and, as such, can be written at date $t$ as:

$$
R_{t}=\frac{p_{t}}{f_{t}\left[p_{t}, p_{t-1}, \ldots, p_{t-n}\right]}
$$

where $f_{t}$ is a function of new cases from date $t$ to date $t-n$, which can be thought of as the infectious potential, that is, the average number of people who have been infected at $t$ and before, and who can infect people at $t$. The parameter $n$ is related to infection duration. Specifications for $f_{t}$ have been used in the literature. For example, Fraser's [11] equation (9), on page 3 of his paper, defines the time-varying effective reproduction number as follows:

$$
R_{t}=\frac{p_{t}}{\sum_{j=0}^{n} w_{j} p_{t-j}}
$$

where the weights $w$ 's capture the generation time distribution, with $\sum_{j=0}^{n} w_{j}=1$. This means that the time-independent function $f$ that follows from the denominator in equation (3) is, in that case, assumed to be linear in the number of cases $p$ (which does not imply that $p_{t}$ is linear in time of course). Note that such an assumption implies that, given the reproduction number $R$, the dynamics of new cases follow an auto-regressive process $A R(n)$. 
Even though it might go unnoticed a first sight, we should stress that a major difference between the rather general definition of $R$ in equation (3) and the usual definition in equation (4) is that the function $f_{t}$ implicitly depends on calendar time $t$. That is, what is taken account of are simply the number of cases detected on a particular day, but not the fact that the number of cases detected will depend on the diagnostic effort or number of tests that has been realized. The fact that the diagnostics dimension is largely ignored in the literature about SIR-type models surfaces, for instance, in Wallinga and Lipsitch [21], who relate the epidemic growth rate to incidence and generation time interval only. It seems reasonable to assume that infectious and emerging diseases involve a diversity of pathogens, which require a variety of technologies to diagnose. In the context of COVID-19, PCR and antigen testing is of course key. This difference in accounting for cases turns out to be important to understand the connection between the acceleration index and the reproduction number, as we now show.

Using equations (2) and (3), we can relate our acceleration index and the reproduction number in the following way:

$$
\varepsilon_{T}=R_{T} \times \frac{A_{T}}{B_{T}} \quad \text { with } \quad A_{T}=\frac{f_{T}\left[p_{T}, p_{T-1}, \ldots, p_{T-n}\right]}{P_{T}} \quad \text { and } \quad B_{T}=\frac{d_{T}}{D_{T}}
$$

Equation (5) shows that the ratio between the acceleration index and the basic reproduction number can be thought of itself as a ratio. The numerator of this latter ratio, $A$, can be thought of as the current infectivity contribution to cumulated cases, that is, the ratio of the average number of primary cases up to period $T$ who can originate infections in $T$ as a fraction of the total number of persons who have been infected since the outset of the pandemic. The denominator, $B$, on the other hand, represents the fraction of tests in period $T$ out of cumulated tests up to $T$, that is, the current test contribution to cumulated tests. To sum up, the ratio of the acceleration index to the reproduction number is, in any period, the ratio of the infectivity contribution to the test contribution.

From Equation (5) we see that both indicators are equivalent, that is, $\varepsilon=R$, if and only if at all dates $t$ :

$$
f_{t}\left[p_{t}, p_{t-1}, \ldots, p_{t-n}\right]=d_{t} \times \frac{P_{t}}{D_{t}}
$$

Equation (6) is very important to conceptualize the core idea of this paper: in order to properly control for the (time-varying) volume of tests/diagnostics, one needs to use the appropriate function $f_{t}$, that is, one which depends on calendar time because tests do. Said differently, the function $f_{t}$ should be specified in such a way that it takes account of the fact that cases are produced by tests or any other diagnostics. The linear form with no time dependence which appears in the denominator of equation (4) is therefore problematic, as it assumes away tests which are however key to measure the pandemic's dynamics. In this sense, the acceleration index $\varepsilon$ nests the basic reproduction number $R$ : if the function $f_{t}$ is specified as in equation (6), $R$ is equivalent to $\varepsilon$ as it takes account of testing; in any other case, $\varepsilon$ is more general than $R$ as in equation (4) that takes account of cases only. 
To better understand the relation between $R$ and $\varepsilon$ as indicated in equation (5), a more theoretical analysis and its implications may be helpful. First of all, as it also becomes clear from equation (5), when $A=B$ - that is when equation (6) holds - then $\varepsilon=R$. This basically means that if the test contribution $B$ follows the dynamics of the infectivity contribution $A$, there is enough testing to capture viral activity. In fact, seen from this perspective, we have a clear testing strategy: the daily tests $d_{t}$ need to offset the assumed infectiousness captured by function $f_{t}$, and more specifically equation (6). The smaller the total number of cases, the easier it will be to match that testing requirement in particular through contact tracing. As total cases go up, contact tracing and sufficient testing may come to its structural and systemic limits. This in itself is a sign that additional health policies will need to be promoted.

If $A>B$, then $\varepsilon>R$, whilst when $A<B, \varepsilon<R$. In the former case, the infectivity rate $A$ of the pandemic cannot be captured by the test contribution $B$ in place. That is, $R$ does not give the appropriate picture of the infectiousness of the pandemic, in fact it underestimates it. To alleviate this bias, either testing would need to be increased, or viral spread would need to be cut by establishing policies that reduce contacts or a mixture of both. In any case, it shows that equation (3) that composes $R$ does depend on more than past and current cases, because they themselves depend on tests and other factors that may favour or not transmissibility. Equivalently in the latter case, $R$ will overestimate the speed of the pandemic if the test contribution $B$ is greater than the infectivity contribution $A$. In such a situation, greater testing than underlying infectivity will necessarily find more cases, actually too many to reflect the correct transmissibility. To capture the correct picture, either testing would need to be reduced, which however seems counterproductive at least to the extent that testing is a way to look at the underlying viral dynamics, or the infectivity function $f$ of equation (3) needs to be adapted to reflect reduced transmissibility.

A further analysis of equation (5) requires structural assumptions, in particular to generate predictions about how both $\varepsilon$ and $R$, rather than their ratio, move over time. Just for the sake of illustration, we now resort to the handy example of deterministic exponential growth. Time is assumed to be continuous, to ease derivation of results, and the number of cases grows exponentially over time, as usually assumed in epidemiological models, of SIR type and related for example. In such a case, $R$ as defined in equation (4) is constant and any difference between $R$ and $\varepsilon$ is due to differences between $A$ and $B$. For $\varepsilon$, we also have to introduce tests and we assume that they also grow exponentially.

Under those assumptions, we show in Appendix A that while the reproduction number is constant over time, the acceleration index is not, as it features different regimes depending on how the growth rate of daily cases compares with the growth rate of daily tests. For example, when the former is larger than the latter, the acceleration index first rises and then approaches a plateau, where it equals the ratio of growth rates, which is larger than 1 in that case. In contrast, the reproduction number stays constant over time. We can visualize this more easily in the simple setting of exponential growth (see Appendix A), but it also holds more generally that the difference 
Figure 1: Numerical example for exponential growth of both daily cases and daily tests. Panel (a) Acceleration index (blue curve) and Reproduction number (green curve). Panel (b) Infectivity contribution (orange curve) and Test contribution (red curve)

(a) Acc. Index and Rep. Number

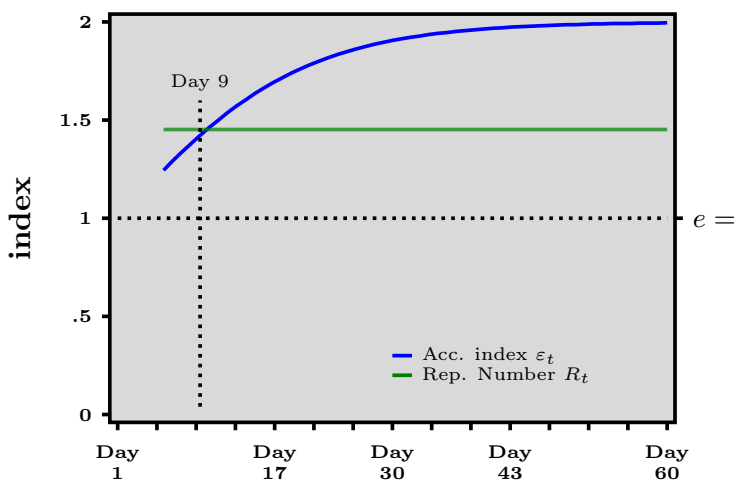

(b) Infectivity and test contributions

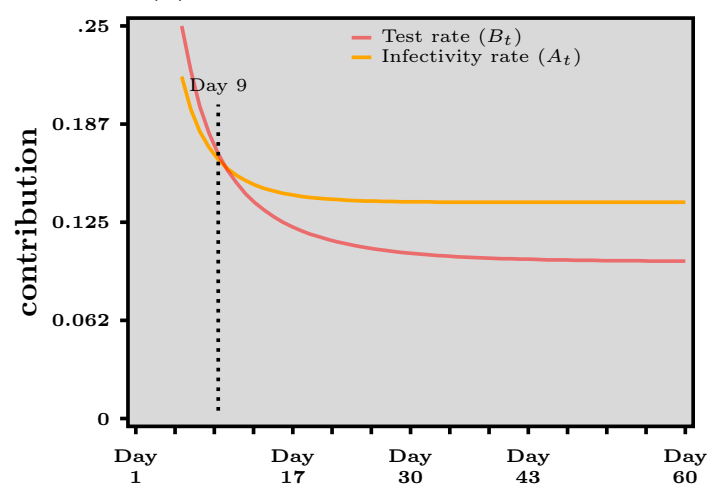

between both indicators is essentially due to the fact that while the acceleration index is the ratio of two growth rates, that of cases divided by that of tests, the reproduction number tracks only the former, thus ignoring the latter. It is also for this reason that we say the the acceleration index $\varepsilon$ nests the basis reproduction number $R$, which is simply a special case of $\varepsilon$. Different configurations may in principle occur, therefore, over time, depending on how fast cases grow compared to the growth of tests.

To further illustrate what happens in the case of exponential growth outlined above and studied in more details in Appendix A, we now provide an illustration such that the growth rate of daily cases is twice as large as the growth rate of daily tests. Figure 1 illustrates how the acceleration index and the reproduction number, as well as the infectivity and test rates, evolve over time in this particular example.

In Figure 1, panel (a), we report the evolution over time of the time-varying acceleration index $\varepsilon(t)$ and the constant reproduction number $R$ that follow from the numerical example. In Appendix A, we show that while $R$ is constant, $\varepsilon$ tends to the ratio of growth rates, which is equal to 2 in the example. As a consequence, a first regime with the reproduction number exceeding the acceleration number happens, followed by a second regime that features the reverse configuration. Not surprisingly, panel $(b)$ in Figure 1 shows that the first regime materializes when $B(t)>A(t)$ - that is the test contribution exceeds the infectivity contribution - while the second regime is associated with $A(t)>B(t)$. Panel $(b)$ reveals in particular that the plateau for the acceleration index that is featured in panel $(a)$ comes from the fact that both $A(t)$ and $B(t)$ themselves plateau, with the infectivity contribution exceeding the test contribution. Overall, therefore, the acceleration tracks the ratio of grow rates - which equals 2 in our example - while the reproduction number underestimates that ratio because it roughly reflects only its numerator.

In the next section, we apply the theoretical decomposition outlined above to capture how the 
medRxiv preprint doi: https://doi.org/10.1101/2020.12.01.20241570; this version posted November 5, 2021. The copyright holder for this preprint (which was not certified by peer review) is the author/funder, who has granted medRxiv a license to display the preprint in It is made available under a CC-BY-NC-ND 4.0 International license.

Figure 2: France - Panel (a) Acceleration index (blue curve) vs reproduction number (green curve). Panel (b) Infectivity contribution (orange curve) vs test contribution (red curve). Panel (c) Kernel estimates with confidence bands (dashed lines). Panel $(d)$ Daily tests (purple line) and infectivity function (black curve). Beige area depicts the lock-down period. Source: Agence Santé Publique France and authors' computations.

(a) Acc. Index and Rep. Number

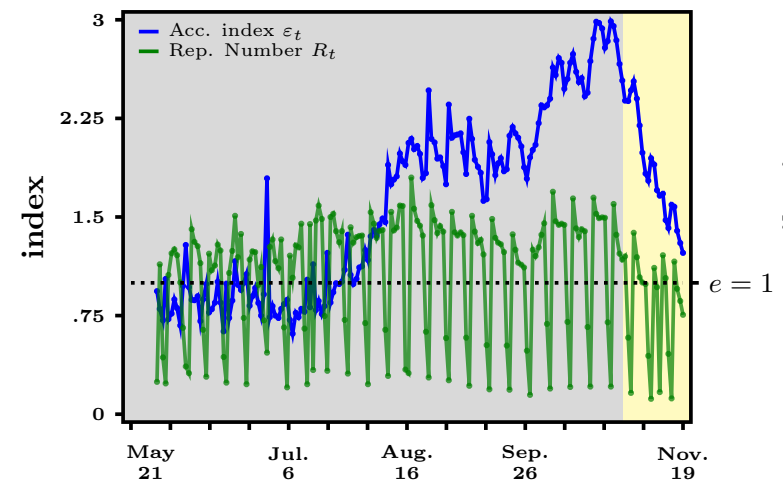

(c) Estimates

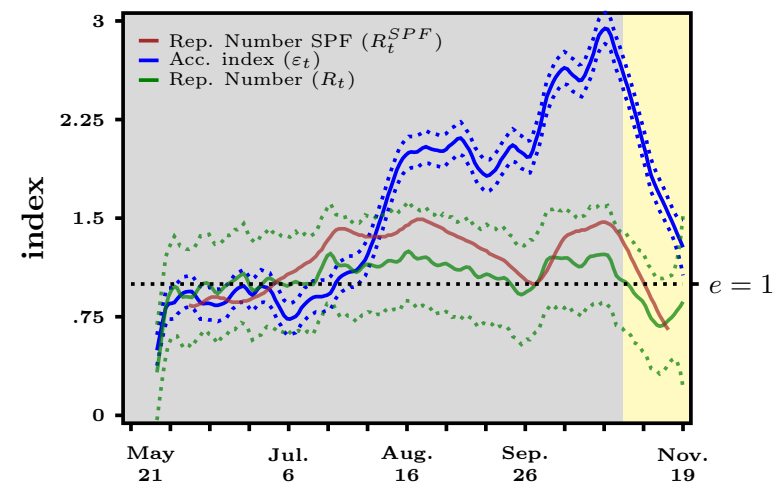

(b) Infectivity and test contributions

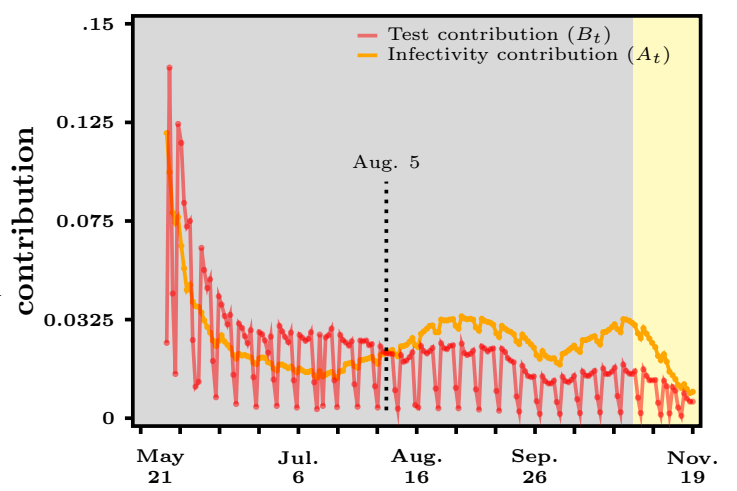

(d) Daily tests and infectivity function

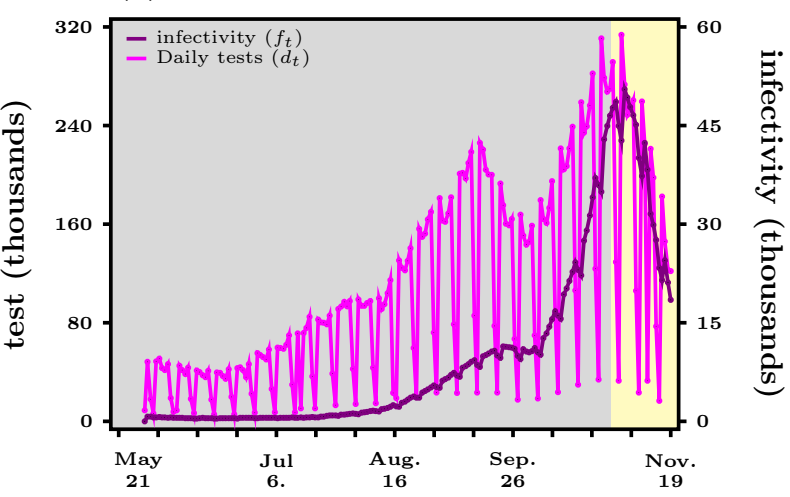

reproduction number and acceleration index differ in the context of the current COVID-19 epidemic in France.

\section{Results and Discussion}

In Figure 2, panel (a), we report both the acceleration index (blue curve) and the reproduction number (green curve) over time, using data for France (May 13 to November 19, 2020). The acceleration index $\varepsilon$ is computed using equation (1) while the reproduction number $R$ is computed from equation (4) with $n=7$ and equal weights $w$. Note that the infection kernel could be adapted to account for sub-exponential growth as in Chowell et al. [8]. The lower spikes of the reproduction number $R$ are due to lower amount of testing during week-ends. This can clearly be seen in the 
panel $(d)$ of Figure 2 that presents the number of daily tests (in pink). We represent here the raw data rather any smoothed estimates in order to avoid any additional layer of interpretation.

In panel (c) of Figure 2, we present local polynomial regressions for $R$ and $\varepsilon$ of panel $(a)$, that use the Savitzky-Golay filter also known as a locally estimated scatter-plot smoothing method in modern statistics (see Cleveland and Devlin [7]). The blue line is again our acceleration indicator. In red, we show the reproduction number as used by Santé Publique France, whereas the green line represents our own estimation. As can be noted, the reproduction number estimated by Santé Publique France falls within the confidence bands of our own estimate which are the dotted green lines. Even though Santé Publique France refers to the Cori method, we have not found public information about the weights attached to past values for the number of cases in computing infectivity. In addition, as can be seen from the confidence bands, the acceleration index is estimated more precisely than the reproduction number, because we take account of variations of tests and thus cases due to the weekend effect. In effect, being a ratio of growth rates (that of cases over that of tests), the acceleration index turns out to be smoother than the reproduction number (which is closer to the growth rate of cases).

If we concentrate on panel (c) of Figure 2, we see that right after the end of the first lock-down, both indicators are roughly "plateauing" just under 1 , with $R$ being slightly above $\varepsilon$. We concentrate here on the green line, i.e. our estimation of $R$. This estimated $R$ then rises quickly to a higher plateau in the first half of July to indicate greater transmissibility, and stays at a level of about 1.2 until mid-August. At that same time $\varepsilon$ first remains put at a level smaller than 1 and becomes greater than 1 a few days later, effectively crossing $R$ at the beginning of August and accelerating all along until about mid-August.

The difference in dynamics of both indicators for France can easily be explained by looking at panel (b) in Figure 2. Here, we report the two terms that appear in equation (5), that is, $A$, the infectivity contribution (orange curve), and $B$, the test contribution (red curve). The latter graph exhibits spikes, due to the fact that much less tests, if any, are performed during week-ends. The test contribution follows a downward trend that simply reveals the fact that tests being done in a given period constitute, over time, a smaller and smaller fraction of the cumulated amount of diagnostics. What we see in panel $(b)$ in particular is that before August 5 , the test contribution $B$ is greater than the infectivity contribution $A$ that has, at first, also a downward trend. A greater testing rate implies that more cases will be found. This corresponds to the period when $R$ is greater than $\varepsilon$. But $R$ basically overestimates viral activity because it does not consider tests and focuses on cases only, while $\varepsilon$ takes account of this because it looks at the ratio of both infectivity and test contributions. The opposite is true for the period after August 5, when the infectivity contribution $A$ becomes greater than the test contribution $B$, and $\varepsilon$ is greater than $R$. Despite growing daily tests until the end of August, as we can see in panel $(d)$ of Figure 2, $R$ remains first at a plateau but then steadily declines until the second half of September. 
This clearly shows that $R$ has been unable to represent viral acceleration basically during both summer months because, as we can see from panel $(b)$, the infectivity contribution is rising more quickly than the test contribution. Therefore, $R$ overlooks the testing dimension and only captures the number of cases, but those are undervalued given a lower test rate $B$. Even worse, testing then declines at the end of August while the infectivity function $f$, indicated in black in panel $(d)$ starts going up. This affects $R$ that declines to reach a level of about 1 by the end of September. This is in very stark contrast to $\varepsilon$ that accelerates from early July onwards and then hovers at a plateau of about 2 up to end of September. It takes appropriately into account the relationship between the changing growth rates of testing and infectivity.

Both indicators go up again from the end of September onwards as testing rises again. But while $R$ reaches a plateau again as the first curfew measures where put into place to cut transmission, $\varepsilon$ further indicates acceleration. Both indicators then start declining when, at the end of October, the second lock-down was put into place.

However, $R<1$ since the beginning of November, whilst at the same time, our indicator $\varepsilon$ still shows an ongoing acceleration, although a reduced one with respect to the time before the lockdown. What we see very clearly from panel $(d)$ is that lock-down coincides with a great reduction of testing. Obviously, lock-down is aimed at reducing contacts and thus viral spread. This will necessarily reduce cases and hence $R$ declines. But if at the same time testing is reduced as well, which is the only way to get a clearer picture of the viral activity this necessarily influences $R$ more dramatically and explains the under-evaluation of the viral spread than $\varepsilon$ that continues to indicate acceleration. More specifically, $\varepsilon$ indicates what happens to cases when we reduce testing by some percentage change. The fact that the percentage change of cases goes in the same direction as the percentage change of testing, i.e. that both decrease, is a good sign and indicates that lock-down measures have their effect. But looking at $\varepsilon$ does not yet allow to give an all-clear such as $R$ does. As a consequence, $\varepsilon$ captures more accurately the considerable time variation of virus propagation than $R$.

To make clear that the discrepancy between the reproduction number and the acceleration index shown in Figure 2 is not specific to France, we report in Appendix B a similar decomposition for five other countries. Such a comparison reveals that the reproduction number might either underestimate or underestimate viral acceleration, depending on the country and the time period, due to not correcting for the time-varying amount of testing. This readily suggests that this issue might be highly relevant in many other countries as well, where public health authorities are also in dire need of accurate indicators to track epidemics. As such, this observation should also preoccupy international bodies that design cooperation strategies to fight pandemics, including of course the World Health Organization and other regional agencies.

To futher illustrate the differences between $R$ and $\varepsilon$ and their consequences for public health decisions, we consider Figure 3. We use a background map that is in the public domain and can be down- 
medRxiv preprint doi: https://doi.org/10.1101/2020.12.01.20241570; this version posted November 5, 2021. The copyright holder for this preprint (which was not certified by peer review) is the author/funder, who has granted medRxiv a license to display the preprint in

It is made available under a CC-BY-NC-ND 4.0 International license .

Figure 3: Acceleration index and reproduction number for French départements at different dates. October 30 dates the start of the second lock-down in France. Data source: Agence Santé Publique France and authors' computations.

\section{REPRODUCTION NUMBER $R$}

(a) Oct. 30

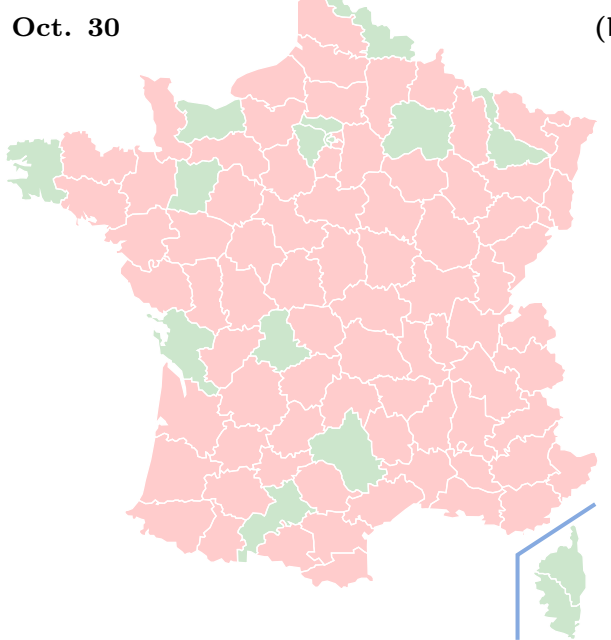

(b) Nov. 19

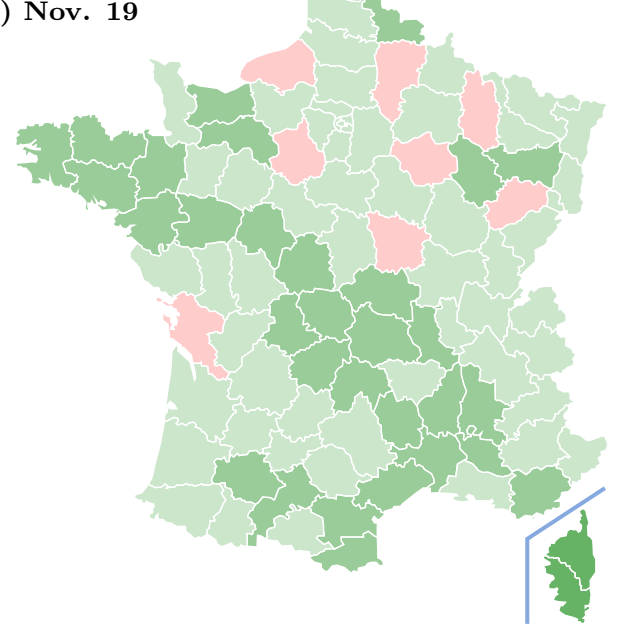

Reproduction number

More than 5

between 4 and 5

between 3 and 4

between 2 and 3

between 1 and 2

between 0.8 and 1

between 0.6 and 0.8

between 0.4 and 0.6

between 0.2 and 0.

between 0 and 0.2

\section{ACCeleration INDEX $\varepsilon$}

(c) Oct. 30

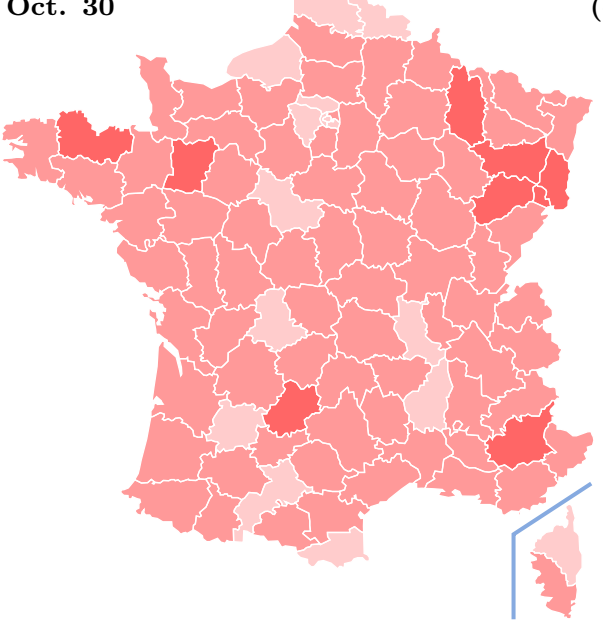

(d) Nov. 19

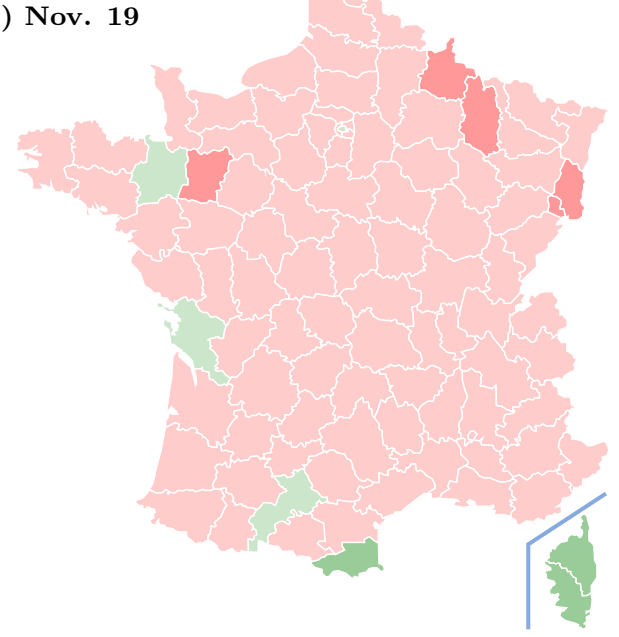

Acceleration index

More than 5

between 4 and 5

between 3 and 4

between 2 and 3

between 1 and 2

between 0.8 and 1

between 0.6 and 0.8

between 0.4 and 0.6

between 0.2 and 0.4

between 0 and 0.2

loaded via https://commons.wikimedia.org/wiki/File:D\%C3\%A9partements_de_France-simple.

svg. Figure 3 is then constructed by incorporating our own data into this background map and gives an overview of how the second lock-down, which started on October 30, 2020, has contributed to reduce virus circulation across French départements. Looking at the two bottom maps shows that the acceleration index has been reduced everywhere during the period from October 30 to November 19. While a similar improvement is indicated by the reproduction number, as one concludes from the top maps, that measure of virus spread tells an altogether different story. Three weeks after the beginning of the second lock-down in France, the acceleration index suggests that 
the acceleration regime still prevails, except for 6 départements which happen to be the happy few, but with values still close to unity. However, one concludes rather wrongly from the reproduction number that, at the same date, deceleration is underway in most départements in green. In view of the discussion about the $\varepsilon / R$ ratio in Section 3, this comes as no surprise since the reproduction number under-estimates virus circulation, due to the fact that it does not take tests into account. To sum up, two main differences between the reproduction number and the index appear in panel (c). The first being that $R$ crosses unity earlier than the acceleration index, which starts to increase around July 6 . This is most likely due to the infectivity rate reaching its lowest point around that date (as seen in panel $(b)$ ). Passing that date, the infectivity rate begins to increase, while the test rate continues downwards. This explains why the acceleration index could not start growing before July 6. Overall, therefore, $R$ is larger than $\varepsilon$ before August 5. The second difference is a sudden decrease of the reproduction number in the second half of September, while the acceleration index stays at a plateau. This can be explained by panel $(d)$, in which we see a sharp plummet in the number of daily tests around that period. Less tests equals less detected cases, which $R$ relies heavily on for its calculation. Seeing $R$ rising sharply from about 1 in early October is all the more surprising when seen in isolation. In contrast, the acceleration index, which accounts for variations of both cases and tests, consistently shows a succession of periods of steep rise followed by plateaus over the summer and until the second lock-down.

In practice, many public health agencies report (daily or weekly) positivity rates, to complement the information contained in the reproduction rate $R$. In light of the connection with $\varepsilon$ that we have highlighted in this note, both formally and empirically, we argue that the acceleration index is closer to a sufficient statistic that helps tracking the rapidly changing dynamics of any pandemic, because it explicitly takes into account the dynamics of diagnostics. In the context of COVID-19, diagnostics equal tests, but our claim is valid more generally when this is not the case. This means that the index can potentially be applied to any effort designed at detecting infected people, no matter what the pathogen agent triggering the infectious disease turns out to be. In real time, this is quite valuable, we believe, to guide health policies and to assess containment measures, especially in the context of a new pathogen appearing (such as SARS-Cov-2), with unforeseeable pandemic dynamics.

\section{Conclusion}

We show in this paper that the reproduction number $R$ is a special case of the the acceleration index proposed in Baunez et al. [5]. While the former only considers the growth rate of cases, the latter measures variations of cases in relation to that of tests, and it does so in a unit-free manner since it is an elasticity. Most importantly, the acceleration index is a sufficient statistic of viral spread in the sense that it aggregates all the relevant information in a synthetic manner. In contrast, looking 
at pieces of information, like positivity or prevalence rates, separately might lead to the misleading conclusion that there is conflicting evidence about whether the epidemic worsens or improves. As such, a test-controlled reproduction number like the acceleration index should be part of any data dashboard to track an epidemic, and especially to guide public policy in the design of the most efficient methods to curb it. For example, we have shown in Baunez et al [5] that an accurate measure of virus circulation is a key input to feed algorithms that are designed to efficiently allocate the diagnostic effort across space.

The result that the reproduction number is, as a measure of viral spread, subject to a considerable bias is not specific either to France or to the period considered, as illustrated using data from five other countries. Such a comparison reveals that the reproduction number might either underestimate or underestimate viral acceleration, depending on the country and the time period, due to not correcting for the time-varying amount of testing. This readily suggests that this issue might be highly relevant in many other countries as well, where public health authorities are also in dire need of accurate indicators to track epidemics. As such, this observation should also preoccupy international bodies that design cooperation strategies to fight pandemics, including of course the World Health Organization and other regional agencies.

Such an observation makes the acceleration index a much more useful indicator to track a pandemic in real-time, as it is context-dependent: the acceleration index takes into account the effort to diagnose people who have been infected by the pathogen. In the case of COVID-19, diagnostics equal PCR (and other types of biological) tests, but this might not be the case for other diseases where diagnostics require even greater effort. However, our analysis makes a strong case for incorporating in any measure of pathogen circulation the observed effort to diagnose the agent that makes people sick.

Even though there is a variety of infectious (and emerging) diseases, with different pathogens and various ways to diagnose them, we claim that our conceptual approach is general enough to shed light, not only on the current pandemic, but also on any future ones which may come.

Finally, we would like to mention some limitations of our analysis. Some important issues, beyond the scope of our paper, have however been addressed by the literature. First, the fact that unaccounted cases arise when testing is not compulsory (see Pullano, Di Domenico, Sabbatini et al. [18] for France). Second, the coexistence of symptomatic and asymptotic cases during COVID-19 has led to additional statistical methods (see Khailaie et al. [15]).

\section{References}

[1] Althaus C.L. (2014): Estimating the Reproduction Number of Ebola Virus (EVOB) during the 2014 Outbreak in West Africa. PLOS Currents, Sep 2:6. 3 
medRxiv preprint doi: https://doi.org/10.1101/2020.12.01.20241570; this version posted November 5, 2021. The copyright holder for this preprint (which was not certified by peer review) is the author/funder, who has granted medRxiv a license to display the preprint in It is made available under a CC-BY-NC-ND 4.0 International license .

[2] Bauch C.T., Lloyd-Smith J.O., Coffee M.P., Galvani A.P. (2005): Dynamically Modeling SARS and Other Newly Emerging Respiratory Illnesses: Past, Present, and Future. Epidemiology, 16:791-801. 3

[3] Baunez C., Degoulet M., Luchini S., Pintus P., Teschl M. (2020): Sub-National Allocation of COVID-19 Tests: An Efficiency Criterion with an Application to Italian Regions. Covid Economics, 12:192-209.

[4] Baunez C., Degoulet M., Luchini S., Pintus P., Teschl M. (2020): An Early Assessment of Curfew and Second COVID-19 Lock-down on Virus Propagationin France. MedR $\chi$ iv preprint 11.11.20230243 available at https://doi.org/10.1101/2020.11.11.20230243. 2, 4

[5] Baunez C., Degoulet M., Luchini S., Pintus P., Teschl M. (2021): Tracking the Dynamics and Allocating Tests for COVID-19 in Real-Time: an Acceleration Index with an Application to French Age Groups and Départements. PLoS ONE, June 1st, available at https://doi.org/ 10.1371/journal.pone.0252443. 2, 4, 13, 14

[6] Baunez C., Degoulet M., Luchini S., Pintus P., Teschl M. (2021): COVID-19 Acceleration and Vaccine Status in France - Summer 2021. MedR $\chi$ iv preprint 11.11.20230243 available at https://www.medrxiv.org/content/10.1101/2021.09.18.21263773v3. 2

[7] Cleveland, W.S., Devlin, S.J. (1988): Locally-Weighted Regression: An Approach to Regression Analysis by Local Fitting. Journal of the American Statistical Association. 83:596-610. 10

[8] Chowell G., Viboud C., Simonsen L., Moghadas S.M. (2016): Characterizing the Reproduction Number of Epidemics with Early Subexponential Growth Dynamics. Journal of the Royal Society Interface, 13(123):20160659. 9

[9] Cori A., Ferguson N.M., Fraser C., Cauchemez S. (2013): A New Framework and Software to Estimate Time-Varying Reproduction Numbers during Epidemics. American Journal of Epidemiology, 178:1505-1512. 2, 4

[10] Cori A., Donnelly C.A., Dorigatti I., Ferguson N.M., Fraser C., Garske T., Jombart T., NedjatiGilani G., Nouvellet P., Riley S., Van Kerkhove M.D., Mills H.L., Blake I.M. (2017): Key Data for Outbreak Evaluation: building on the Ebola Experience. Philos Trans $R$ Soc Lond B Biol Sci. May 26; 372 (1721):20160371. 3

[11] Fraser C. (2007). Estimating Individual and Household Reproduction Numbers in an Emerging Epidemic. PLoS One, 2:e758. 2, 4, 5

[12] Kermack W.O., McKendrick A. G. (1927): A Contribution to the Mathematical Theory of Epidemics. Proceedings of the Royal Society of London. Series A, 115:700-721. 3 
medRxiv preprint doi: https://doi.org/10.1101/2020.12.01.20241570; this version posted November 5, 2021. The copyright holder for this preprint (which was not certified by peer review) is the author/funder, who has granted medRxiv a license to display the preprint in It is made available under a CC-BY-NC-ND 4.0 International license .

[13] Kermack W.O., McKendrick A. G. (1932): A Contribution to the Mathematical Theory of Epidemics II. The Problem of Endemicity. Proceedings of the Royal Society of London. Series A, 138:55-83. 3

[14] Kermack W.O., McKendrick A. G. (1933): A Contribution to the Mathematical Theory of Epidemics. III. Further Studies of the Problem of Endemicity. Proceedings of the Royal Society of London Series A, 141: 94-122. 3

[15] Khailaie, S., et al. (2021): Development of the reproduction number from coronavirus SARSCoV-2 case data in Germany and implications for political measures. BMC Med, 19:32. 14

[16] Marshall, A. (2013[1890]): Principles of Economics. Palgrave Macmillan. ISBN 978 - 0 - 230 $24929-5.4$

[17] May R.M., Anderson R.M. (1991): Infectious Diseases of Humans: Dynamics and Control. Oxford University Press. ISBN $0-19-854040-X .2,3$

[18] Pullano, G., Di Domenico, L., Sabbatini, C.E. et al. (2021): Underdetection of Cases of COVID19 in France threatens Epidemic Control. Nature, 590: 134â139. 14

[19] Ritchie, H., Mathieu, E. Rodés-Guirao, L., Appel, C., Giattino, C., Ortiz-Ospina, E., Hasell, J., Macdonald, B., Beltekian, D., Roser, M. (2020): Coronavirus Pandemic (COVID-19). Published online at OurWorldInData.org. Retrieved from: https://ourworldindata.org/coronavirus [Online Resource] 19

[20] Viceconte G., Petrosillo N. (2020): COVID-19 R0: Magic Number or Conundrum? Infectious Disease Reports, 12:8516. 3

[21] Wallinga J., Lipsitch M. (2007): How Generation Intervals Shape the Relationship between Growth Rates and Reproductive Numbers. Proceedings of the Royal Society of London Series B, 274:599-604. 6

[22] Weiss H. (2013): The SIR model and the Foundations of Public Health. MATerials MATemátics, Volume 2013, no. 3, 17 pp. ISSN: $1887-1097.3$ 


\section{A Exponential Growth: an Illustrative Example}

We now illustrate the relationship between the acceleration index and the reproduction number when time is assumed, to ease derivation of results, to be continuous and when the number of cases grows exponentially over time, as usually assumed in epidemiological models, of SIR type and related for example. Although typically absent in the latter strand of literature, we have to introduce tests and we assume that they also grow exponentially. More formally, using the notation in the previous section, suppose that the number of cases per unit of time is denoted by $p(t)=\alpha e^{\beta t}$ while the number of tests per unit of time is $d(t)=\gamma e^{\nu t}$, where the growth rates $\beta$ and $\nu$ are assumed to be positive for the sake of illustration. The reproduction number is then constant over time, as we now show when the infection kernel is uniform, that is, if the weights $w$ are constant and equal to $1 / \delta$ to ensure that $w \int_{0}^{\delta} d s=1$. Note that it is not difficult to show that the constancy of $R$ holds for other infection distributions as well. The analog of equation (4) is as follows:

$$
R(t)=\frac{p(t)}{w \int_{0}^{\delta} p(t-s) d s}
$$

which implies that, given $p(t)=\alpha e^{\beta t}$, one has:

$$
R(t)=R=\frac{\beta \delta}{1-e^{-\beta \delta}}
$$

It is easily seen that the reproduction is the growth rate of daily cases (adjusted for the delay $\delta$ ) only, since it obviously does not take into account tests. In fact, $R$ is the growth rate of the denominator in equation (7), that is, what we note $f$ in the main text.

Cumulated cases and tests are then noted $P(t)=\int_{0}^{t} p(\tau) d \tau$ and $D(t)=\int_{0}^{t} d(\tau) d \tau$, respectively. It is easy to derive, by straight integration, the expressions:

$$
P(t)=\frac{\alpha}{\beta}\left(e^{\beta t}-1\right), D(t)=\frac{\gamma}{\nu}\left(e^{\nu t}-1\right)
$$

It follows that our acceleration index is given, as function of time, by:

$$
\varepsilon(t)=\frac{p(t) / P(t)}{d(t) / D(t)}=\frac{\beta}{\nu}\left(\frac{1-e^{-\nu t}}{1-e^{-\beta t}}\right)
$$

From equation (10), one concludes that the acceleration index, which is an elasticity that measures the responsiveness of cases to tests, is essentially the ratio of the growth rate of cumulated cases divided by that of cumulated tests, since $p(t)=d P(t) / d t$ and $d(t)=d D(t) / d t$. In addition, $\varepsilon$ tends to the ratio of the growth rate of daily cases to that of daily cases $\beta / \nu$. This means that the acceleration index tracks that ratio over time and converges to it eventually.

It follows that three cases occur. When $\beta=\nu$, that is, when both daily cases and daily tests grow at the exact same rate, then our acceleration index equals 1 at all dates. When the two growth rates differ, however, $\varepsilon(t)$ converges, when $t$ goes to infinity, to the ratio of growth rates $\beta / \nu$, independently of the scale parameters $\alpha$ and $\gamma$. As an illustrative example, suppose that $\beta>\nu$, so that positives grow faster than tests. Then the pattern of our acceleration index $\varepsilon(t)$ over time will 
have two regimes: it first grows almost linearly and eventually reaches the upper bound $\beta / \nu>1$. Obviously, in that case both the daily positivity rate $p(t) / d(t)$ and the average positivity $P(t) / D(t)$ grow over time, and the latter quantity exceeds the former all the time so that acceleration prevails. The symmetric case when $\beta<\nu$ is easily adapted.

The property that the reproduction number is constant under our assumptions essentially means that the dynamics of the acceleration index is driven by that of $A(t) / B(t)$, which we now decompose to help understand how $R$ and $\varepsilon$ compare over time. It follows from the definition of $A$ and $B$ in equation (5) that:

$$
A(t)=\frac{e^{\beta t}-e^{\beta(t-\delta)}}{\delta\left(e^{\beta t}-1\right)} \quad \text { and } \quad B(t)=\nu \frac{e^{\nu t}}{e^{\nu t}-1}
$$

with both functions $A(t)$ and $B(t)$ decreasing with time $t$.

To further illustrate what happens in the theoretical case outlined above with $\beta>\nu$, let us take a numerical example. Suppose that the growth rate of cases $\beta$ equals $20 \%$ while the growth rate of tests $\nu=10 \%$. In addition suppose that the delay parameter $\delta=4$ so that the weight $w=1 / 4$. Figure 1 illustrates how the acceleration index and the reproduction number, as well as the infectivity and test rates, evolve over time in this particular example.

The exponential example is also useful to illustrate the formal relationship between viral speed and the acceleration index. To do that, let us now make an analogy with linear body motions to relate our indicators to speed and acceleration. If one think of the positivity rate as viral speed and define it as $s(t)=\frac{p(t)}{d(t)}$ then straightforward computations give $s(t)=\frac{\alpha}{\gamma} e^{(\beta-\nu) t}$. By analogy, viral acceleration could be defined as the derivative of viral speed, that is, $a(t)=s^{\prime}(t)=\frac{\alpha}{\gamma}(\beta-\nu) e^{(\beta-\nu) t}$. Not surprisingly, then, it turns out that the sign of $a(t)$ indicates acceleration in the sense that a positive sign reveals acceleration (this happens when $\beta>\nu$ since speed goes up over time in that case) while a negative sign reveals deceleration (when $\beta<\nu$ ).

However, a drawback of $a(t)$ as a measure of acceleration is that it is scale-dependent since it depends on scale parameters $\alpha$ and $\nu$, though their ratio. In other words, $a(t)$ depends on the absolute level of speed, that is, of the positivity rate. On the contrary, the acceleration index given by Equation (10) does not and is unit-free, since it is an elasticity. Note that a possible, also unit-free, alternative would be to define acceleration as the semi-elasticity $a(t) / s(t)=\beta-\nu$. However, since it relates the percentage change of positives case to the absolute change of tests, it is arguably less amenable to interpretation than the elasticity. It is easily shown that the semi-elasticity would be obtained if the average positivity rate would alternatively be defined as the average of the daily positivity rates up to date $T$, as opposed to the ratio of number of cumulated cases to the number of cumulated tests as in Equation (2) which leads to the acceleration index as an elasticity. Finally, note that the acceleration index equals one when viral speed stays constant over time. 


\section{B Test-Controlling the Reproduction Number in Practice: Five Countries}

In this appendix, we provide estimates for both the acceleration index and the reproduction number, for Argentina, Austria, South Africa, South Korea and the United Kingdom, using the same method that is applied in Section 3 for France and over the same period (May 13 to November 19, 2020). The public data source for the five countries comes from Our World in Data (Ritchie et al. [19]). The resulting estimates, depicted in Figures 4 to 8 (that are hence directly comparable to Figure 2 for France), unambiguously reveal that the substantial bias due to time-varying tests, which is corrected by the acceleration index but not by the reproduction number, is not specific to France or to a particular period. In fact, the corresponding bias forces the reproduction number to either underestimate (Argentina over the whole period is a case in point) or overestimate viral acceleration, depending on the country and the period considered. This observation suggests that a similar bias should come as no surprise for other countries as well.

Figure 4: Argentina - Panel (a) Acceleration index (blue curve) vs reproduction number (green curve). Panel (b) Infectivity contribution (orange curve) vs test contribution (red curve). Panel (c) Kernel estimates with confidence bands (dashed lines). Panel (d) Daily tests (purple line) and infectivity function (black curve). Source: Our World in Data and authors' computations.

(a) Acc. Index and Rep. Number

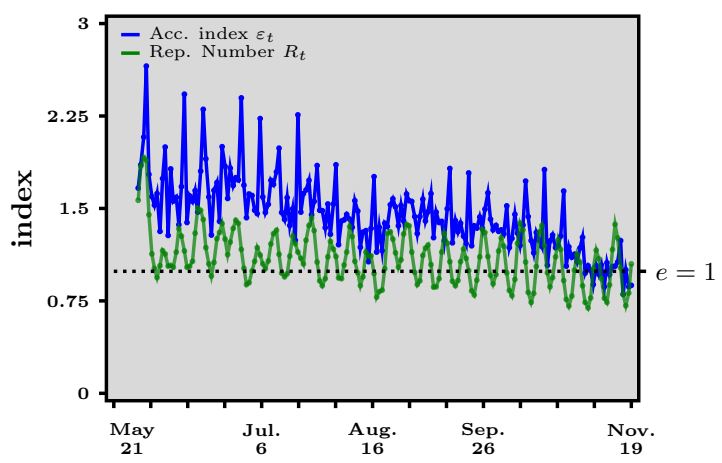

(c) Estimates

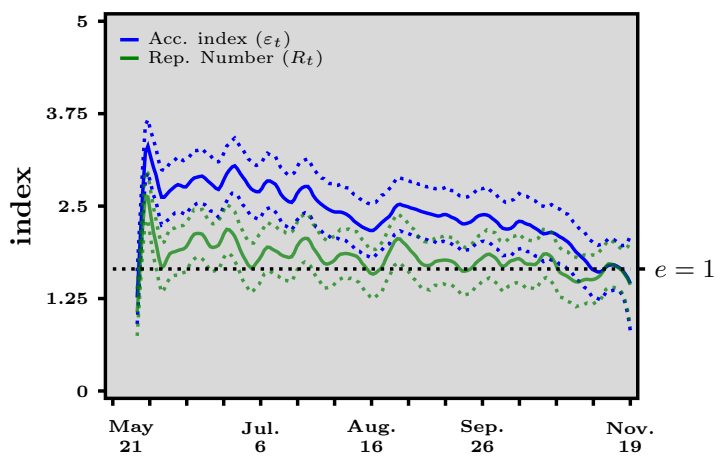

(b) Infectivity and test contributions

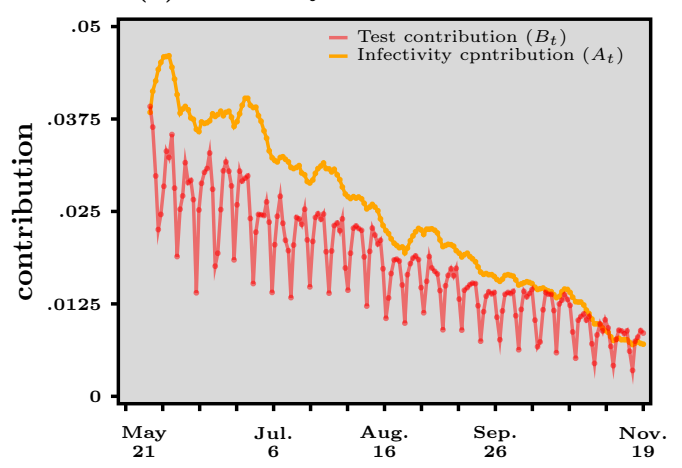

(d) Daily tests and infectivity function

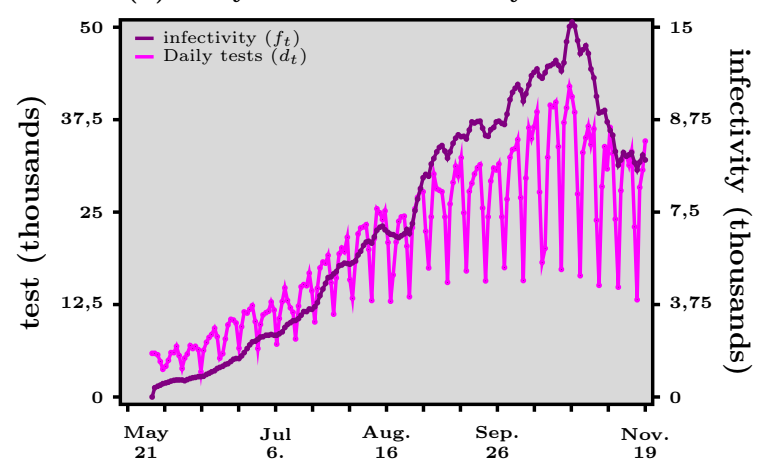


medRxiv preprint doi: https://doi.org/10.1101/2020.12.01.20241570; this version posted November 5, 2021. The copyright holder for this preprint (which was not certified by peer review) is the author/funder, who has granted medRxiv a license to display the preprint in It is made available under a CC-BY-NC-ND 4.0 International license

Figure 5: Austria - Panel (a) Acceleration index (blue curve) vs reproduction number (green curve). Panel (b) Infectivity contribution (orange curve) vs test contribution (red curve). Panel (c) Kernel estimates with confidence bands (dashed lines). Panel $(d)$ Daily tests (purple line) and infectivity function (black curve). Source: Our World in Data and authors' computations.

(a) Acc. Index and Rep. Number

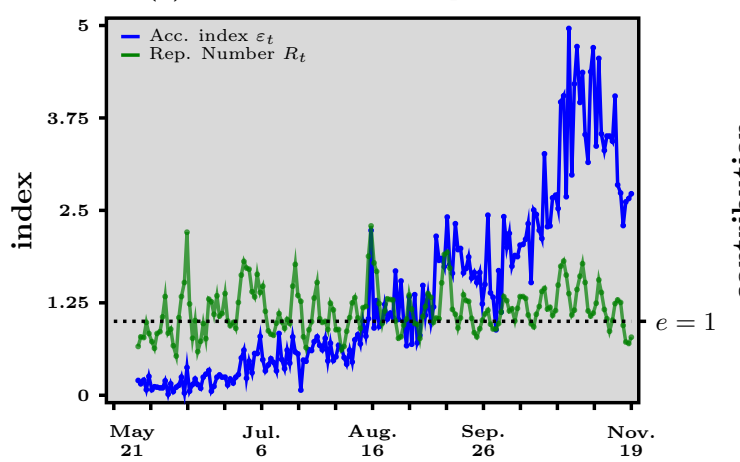

(c) Estimates

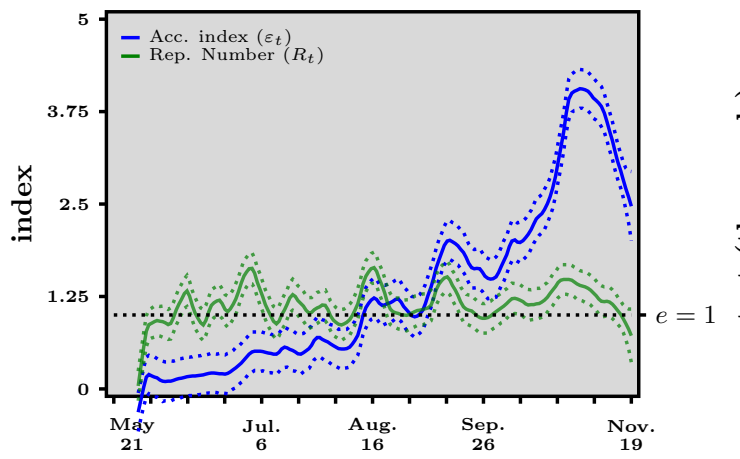

(b) Infectivity and test contributions

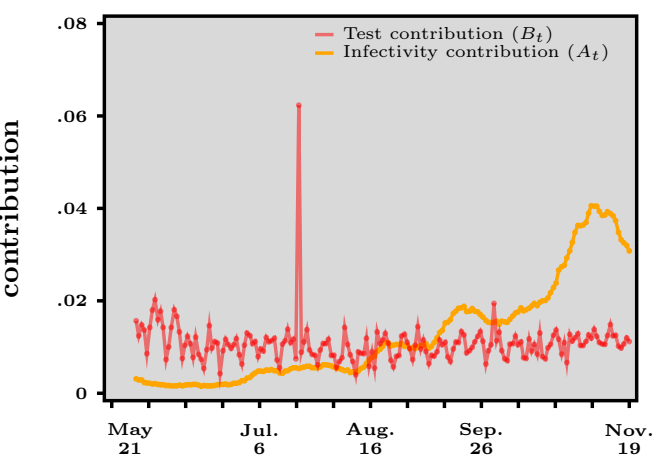

(d) Daily tests and infectivity function

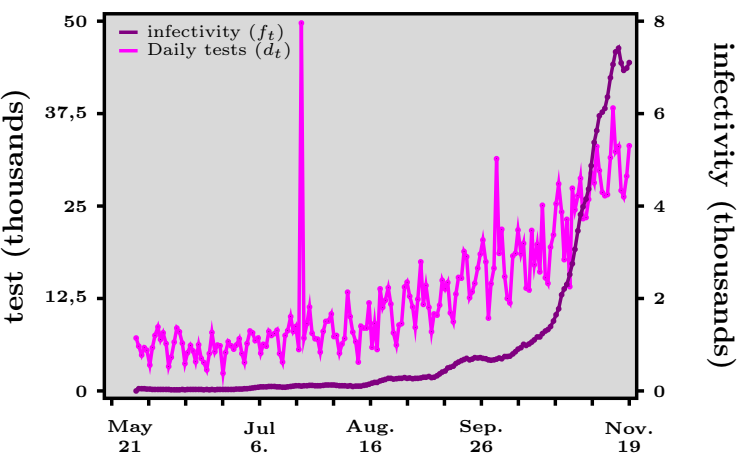


medRxiv preprint doi: https://doi.org/10.1101/2020.12.01.20241570; this version posted November 5 , 2021. The copyright holder for this preprint (which was not certified by peer review) is the author/funder, who has granted medRxiv a license to display the preprint in It is made available under a CC-BY-NC-ND 4.0 International license

Figure 6: South Africa - Panel (a) Acceleration index (blue curve) vs reproduction number (green curve). Panel (b) Infectivity contribution (orange curve) vs test contribution (red curve). Panel (c) Kernel estimates with confidence bands (dashed lines). Panel (d) Daily tests (purple line) and infectivity function (black curve). Source: Our World in Data and authors' computations.

(a) Acc. Index and Rep. Number

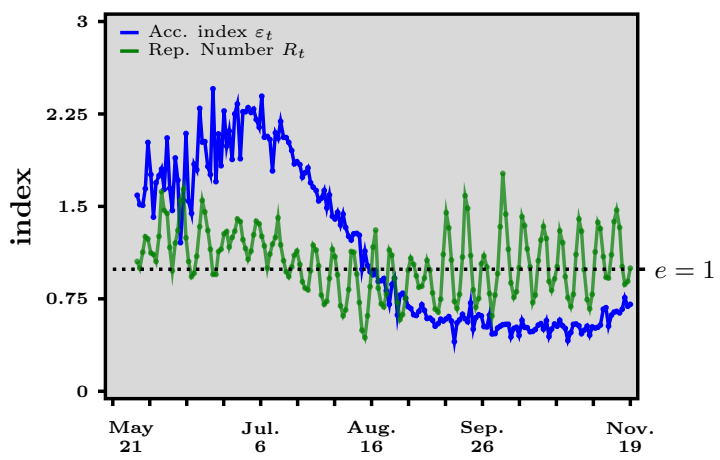

(c) Estimates

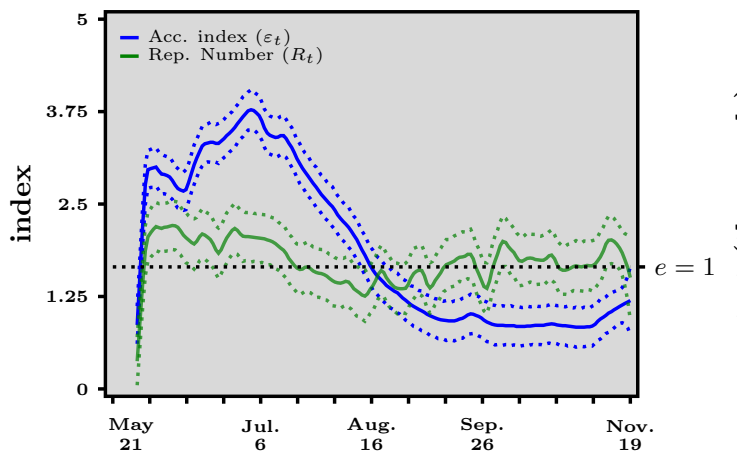

(b) Infectivity and test contributions

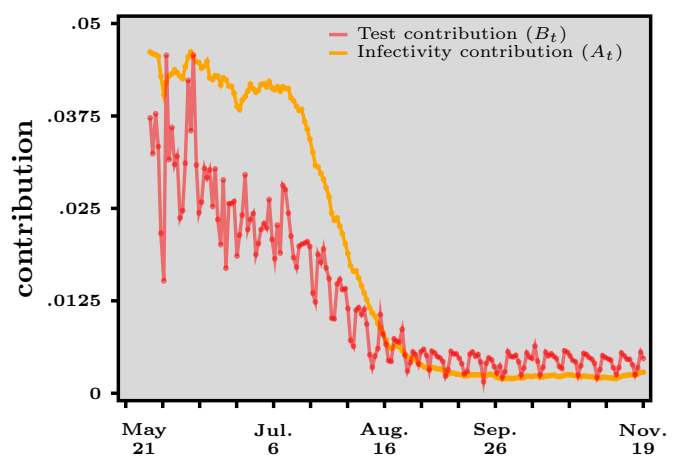

(d) Daily tests and infectivity function

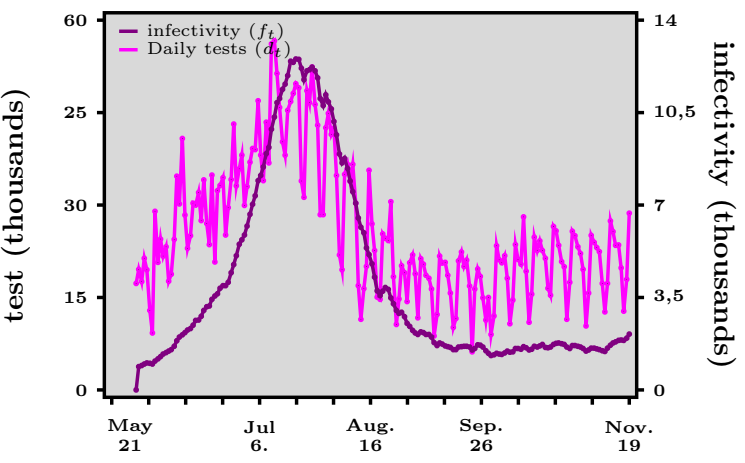


medRxiv preprint doi: https://doi.org/10.1101/2020.12.01.20241570; this version posted November 5, 2021. The copyright holder for this preprint (which was not certified by peer review) is the author/funder, who has granted medRxiv a license to display the preprint in

It is made available under a CC-BY-NC-ND 4.0 International license .

Figure 7: South Korea - Panel (a) Acceleration index (blue curve) vs reproduction number (green curve). Panel (b) Infectivity contribution (orange curve) vs test contribution (red curve). Panel (c) Kernel estimates with confidence bands (dashed lines). Panel (d) Daily tests (purple line) and infectivity function (black curve). Source: Our World in Data and authors' computations. Testing data for South Korea were incomplete: five dates were missing. Missing values were completed by a linear interpolation.

(a) Acc. Index and Rep. Number

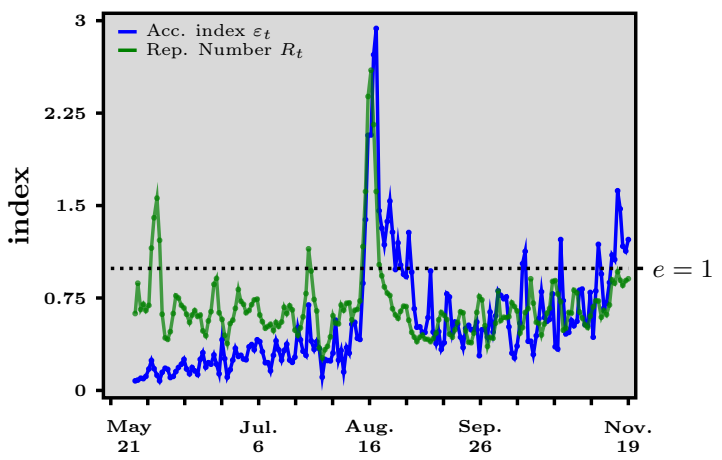

(c) Estimates

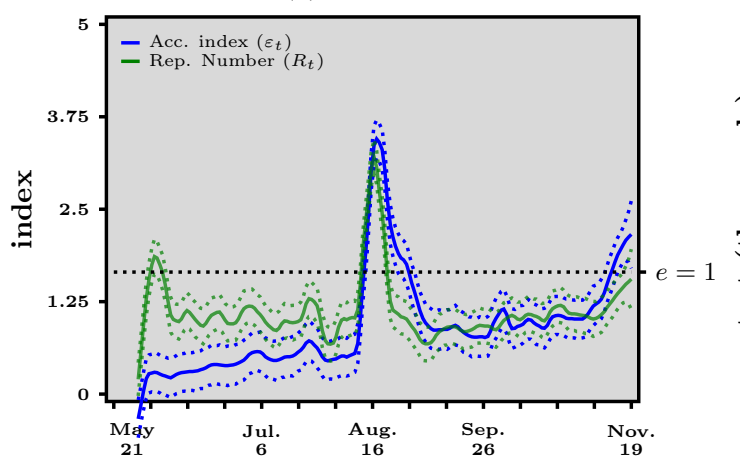

(b) Infectivity and test contributions

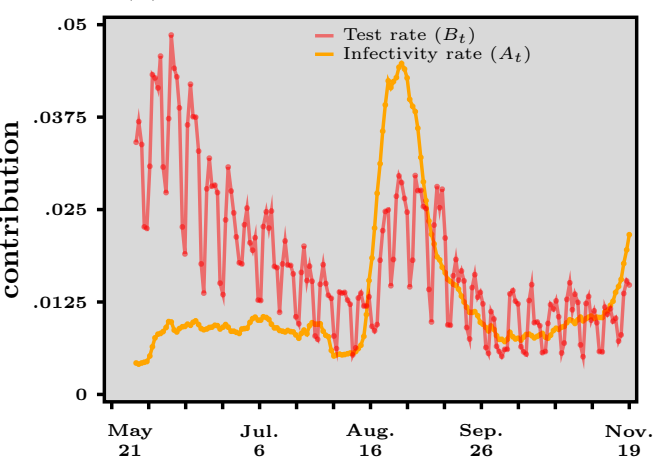

(d) Daily tests and infectivity function

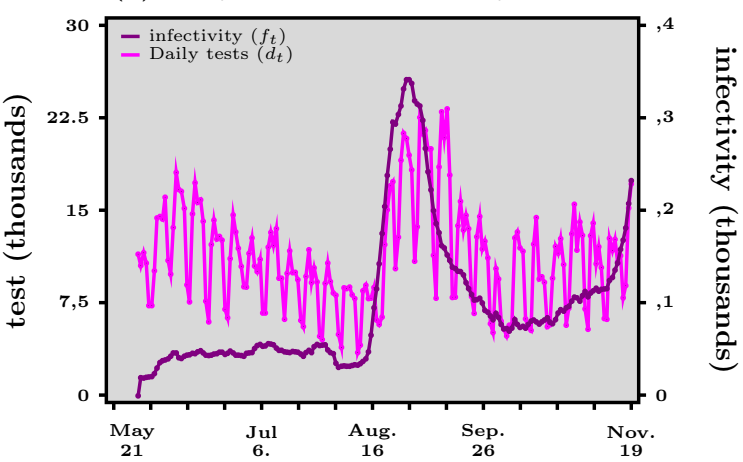


medRxiv preprint doi: https://doi.org/10.1101/2020.12.01.20241570; this version posted November 5, 2021. The copyright holder for this preprint (which was not certified by peer review) is the author/funder, who has granted medRxiv a license to display the preprint in It is made available under a CC-BY-NC-ND 4.0 International license .

Figure 8: United Kingdom - Panel (a) Acceleration index (blue curve) vs reproduction number (green curve). Panel (b) Infectivity contribution (orange curve) vs test contribution (red curve). Panel (c) Kernel estimates with confidence bands (dashed lines). Panel $(d)$ Daily tests (purple line) and infectivity function (black curve). Source: Our World in Data and authors' computations.

(a) Acc. Index and Rep. Number

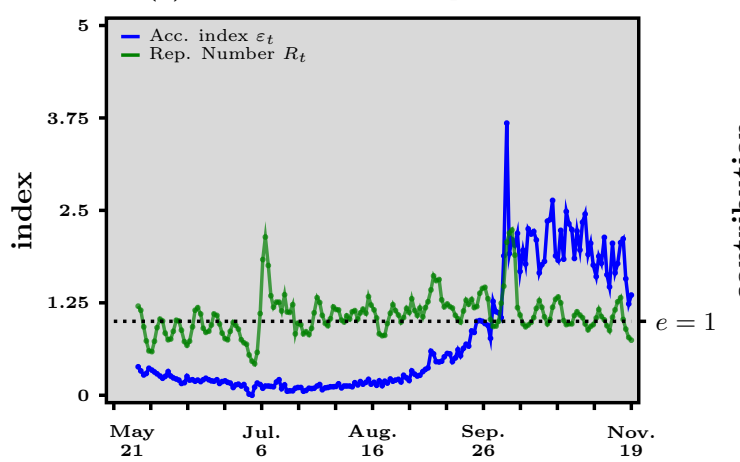

(c) Estimates

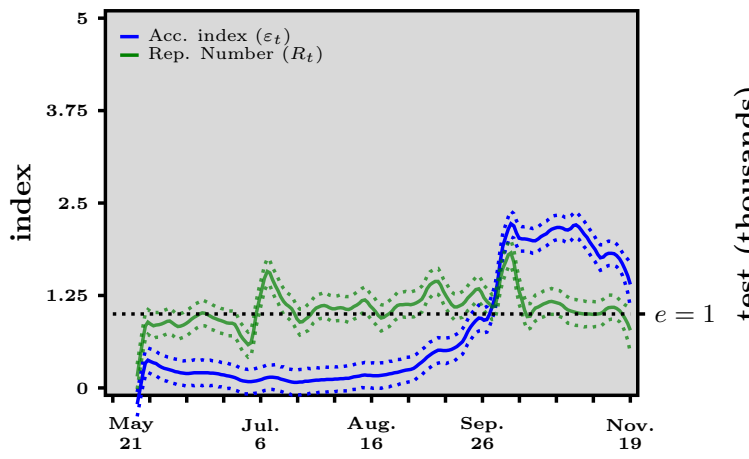

(b) Infectivity and test contributions

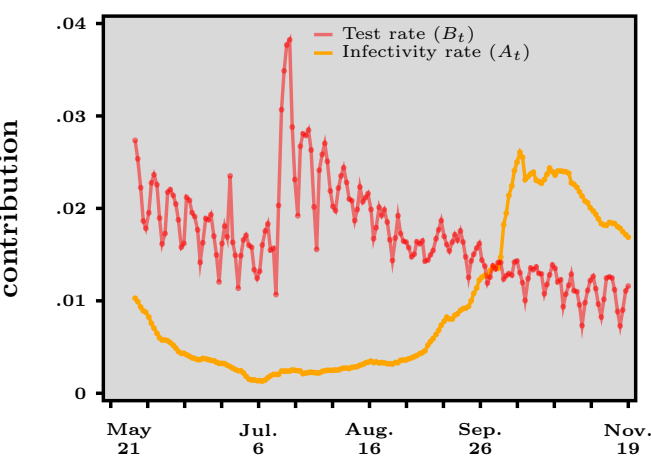

(d) Daily tests and infectivity function

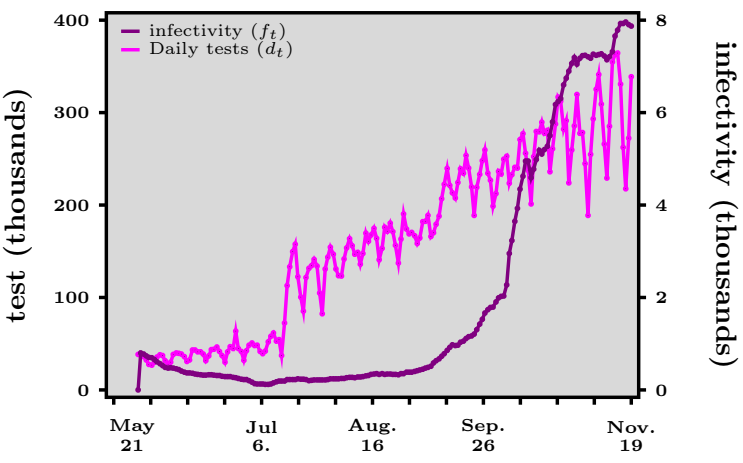

